\title{
Life as a moving fluid: fate of cytoplasmic macromolecules in dynamic fungal syncytia
}

\author{
Marcus Ropera, ChangHwan Lee ${ }^{\mathrm{b}}$, Patrick C. Hickey ${ }^{\mathrm{c}}$, Amy S. Gladfelter ${ }^{\mathrm{d}}$ \\ ${ }^{a}$ Depts. of Mathematics and Biomathematics, University of California, Los Angeles, CA \\ 90095 \\ ${ }^{b}$ Depts. of Biochemistry and Howard Hughes Medical Institute, University of \\ Wisconsin-Madison, WI 53706 \\ ${ }^{c}$ NIPHT Ltd., Edinburgh, EH9 1PL, United Kingdom \\ ${ }^{d}$ Dept. of Biological Sciences, Dartmouth College, Hanover, NH 03755
}

\begin{abstract}
In fungal syncytia dozens, or even millions of nuclei may coexist in a single connected cytoplasm. Recent discoveries have exposed some of the adaptations that enable fungi to marshall these nuclei to produce complex coordinated behaviors, including cell growth, nuclear division, secretion and communication. In addition to shedding light on the principles by which syncytia (including embryos and osteoplasts) are organized, fungal adaptations for dealing with internal genetic diversity and physically dynamic cytoplasm may provide mechanistic insights into how cells generally are carved into different functional compartments. In this review we focus on enumerating the physical constraints associated with maintaining macromolecular distributions within a fluctuating and often flowing cytoplasmic interior.
\end{abstract}

Keywords: Syncytium, mRNA localization, cytoplasmic flow

\section{Introduction}

Biomechanicians have studied extensively the adaptations that enable organisms including plants, insects, larvae and bacteria, to deal with external fluid

\footnotetext{
* Corresponding author

Email address: mroper@math.ucla.edu (Amy S. Gladfelter)
}

Preprint submitted to Current Opinion in Microbiology

June 16, 2015 
flows, from wind to water currents in rivers and the ocean[1]. However cells encounter a parallel challenge of creating and shaping internal flows. Filamentous fungi have especially dynamic cellular interiors: in Neurospora crassa mycelia continuous transport of cytoplasm from the mycelial interior to its growing edges carries nuclei, cytoplasm and organelles at speeds of 10s of microns per second [2]. Even in slower growing fungi nuclei and organelles may be in constant motion $[3,4]$. Although these flows allow long range transport of nutrients and organelles over the colony, mechanisms are needed to localize the mRNAs and proteins that direct spatially regulated processes e.g. cytokinesis [5] or cell-cell communication [6]. In this review we will highlight primarily the role of physical mechanisms that allow ascomycete fungi to localize macromolecules against both diffusion and cytoplasmic flow. We will also discuss how mRNA and protein homogenization between may allow the fungus to tolerate internal genetic and epigenetic diversity. Increasing data suggests that these cellular-level dynamics are a motor for virulence and for the ability of the fungus to adapt to new hosts and changing environments.

\section{Fungal models for dynamic syncytia}

In this review we focus on filamentous ascomycete fungi. There are multiple reasons for choosing filamentous fungi as syncytial models: (1) Fungal compartments are among the largest of syncytia, capable of forming cytoplasmically connected networks that extend several centimeters [7]. (2) Transport within these networks is associated with rapid and long-ranged cytoplasmic flow. For example single nuclei may travel several centimeters through a $N$. crassa mycelium [8] at speeds of up to $60 \mu \mathrm{m} / \mathrm{s}$ [2]. (3). Multinucleate ascomycete cells can be effectively compared with processes that are well studied in the uninucleate yeast S. cerevisiae (4) Filamentous fungi are tolerant of internal genetic diversity; so in some species nuclei can be exchanged by the fusion of genetically distinct mycelia. Although such events are limited (but not absent [7]) in Nature because of somatic recognition mechanisms [6], laboratory-created chimeras allow 
measurement of protein-sharing between nuclei [9]. Note also that although fungal syncytia represent extremes in size and cytoplasmic dynamism; cytoplasmic flow is found in many cells and syncytia, including embryos $[10,11,12]$. Thus syncytial fungi are powerful models to study how macromolecular patterning (Fig 1A) can occur in cells with dynamic cytoplasm.

The heterogeneous patterning of mRNA and protein distributions within fungal syncytia can be inferred from heterogeneous nuclear behaviors and by direct imaging. When synthetic syncytia are created from mammalian, slime mold or yeast cells $[13,14]$, nuclei divide synchronously and so appear to receive identical cues from their shared cytoplasm. However, in fungal syncytia asynchronous nuclear division is common [7]; for example in A. gossypii the sister nuclei produced by the division of a single nucleus divide at the same rate but duplicate DNA and Spindle Bodies at essentially uncorrelated times $[15,16]$. In chimeric fungi genetically different nuclei may be maintained in stably different proportions, suggesting that cyclins can be targeted to specific nuclei within the syncytium $[17,9]$. Protein and mRNA distributions can also be directly imaged using techniques like single molecule Fluorescence in situ Hybridization (smFISH) (Fig. 1A). smFISH shows that in A. gossypii cyclin transcripts form heterogeneous clusters near nuclei [18] and polarity transcripts cluster near incipient hyphal tips [19].

\section{Physical mechanisms for the localization of proteins and mRNAs within the fungal syncytium}

Proteins and mRNAs may travel through a continuous cytoplasm either by diffusion or by flow. In common with other cells [20, 21], filamentous fungi use active transport along the cytoskeleton to localize some mRNAs, for example those associated with polarity establishment [22]. For some proteins (e.g. the protein-rich Spitzenkörper at growing hyphal tips [3]) localization seems to involve protein-cell membrane interactions. However for many macromolecules there is no known cytoskeletal partner or active transport mechanism. Indeed in 
$N$. crassa hypha with fast cytoplasmic flow, microtubules themselves are transported along with cytoplasm (Fig. 1B). Accordingly we focus on cytoskeleton independent mechanisms for patterning macromolecules within the cell. The distribution of any such macromolecule is determined by three physical effects: (i) Diffusion or random thermal motions. (ii) Bulk flow within the flowing cytoplasmic stream. (iii) The lifetime of the macromolecule. For a macromolecule with lifetime $\tau$, diffusivity $D$, and in a cell with cytoplasmic velocity $U$, the relative contributions of diffusion and bulk flow to macromolecular transport can be estimated by comparing the distance that the macromolecule diffuses in its lifetime, $\sqrt{D \tau}$, with the distance it is carried by bulk flow, $U \tau$. The ratio of these two distances is a dimensionless number $C=\sqrt{\frac{D}{U^{2} \tau}}$ : if $C>1$ then most transport of the macromolecule is by the diffusion and if $C<1$, then most transport is by flow. One way to use this criterion is to compute from 75 the diffusivity $D$ and lifetime $\tau$ of a macromoleule the minimum flow velocity at cytoplasmic flow starts to dominate protein distribution. For example for a medium sized macromolecule $\left(D=25 \mu \mathrm{m}^{2} / \mathrm{s}\right)$ with a lifetime $\tau=1 \mathrm{hr}$, cytoplasmic flow dominates diffusion if $U>\sqrt{D / \tau}=0.08 \mu \mathrm{m} / \mathrm{s}$. This is faster than typical velocities within Aspergillus niger hypha $(U=0.04 \mu \mathrm{m} / \mathrm{s}$ [23] $)$ but much lower than velocities seen in $N$. crassa $(U=0.2-60 \mu \mathrm{m} / \mathrm{s})$. By comparison, for a typical-sized mRNA ( $D=0.2 \mu \mathrm{m} / \mathrm{s})$ with the same lifetime, bulk flow is more important than diffusion if $U>0.007 \mu \mathrm{m} / \mathrm{s}$, which is achieved in most growing fungal hyphae [23], To control the distance that macromolecules travel from the sites of their production, a fungal syncytia can manipulate one or more of the physical effects (i)-(iii) (Fig. 1C).

Altering protein diffusion A macromolecule with diffusivity $D$ and halflife $\tau$ will diffuse a distance $\sqrt{D \tau}$ from the site of its production (Fig. 1C). For a cyclin transcript or protein, a useful base of comparison is that in many fungi (including A. gossypii and $N$. crassa), nuclei are spaced around $5 \mu \mathrm{m}$ apart: if 90 a cyclin diffuses this distance or less, it will likely be used by the same nucleus that produces it. Cyclins that diffuse further than $5 \mu \mathrm{m}$ will be shared between multiple nuclei. The diffusivity $D$ can be calculated from the Stokes-Einstein 
relation [24], which states that $D=\frac{k_{B} T}{3 \pi \mu d_{p}}$ where $k_{B} T$ is the thermal energy of the cytoplasm, $\mu$ is the viscosity or stickness of the cytoplasm, and $d_{p}$ is the diameter of the protein. The thermal energy depends only on the absolute temperature $T$ of the cytosol, and varies little between different species (in fact by only $5 \%$ when a species growing at $30^{\circ} \mathrm{C}$ is compared with a species growing at $15^{\circ}$ C.) Accordingly the major variables under fungal control are protein size and viscosity, and we consider adaptation of these parameters separately:

Cytoplasm viscosity The viscosity of cytoplasm varies according to protein content. In mammalian cells, this variation is known to have implications for mRNA dynamics: for example Fusco et al. [25] showed that among $\beta$-actin mRNAs, 30-40\% of transcripts appear to be corralled - i.e. although not stationary, they remained localized to small $\left(<5 \mu \mathrm{m}^{3}\right)$ sub-compartments of the cell - due to interactions between the diffusing mRNAs and the microtubule network. Filamentous fungi also use protein matrices to hinder macromolecular diffusion and it is likely that the physical mechanism is similar. For example the protein Whi3 includes both a polyQ tract and an RNA recognition motif that targets $C L N 3$ message [26]. In A. gossypii, in the presence of $C L N 3$, Whi3 proteins assemble into demixed liquid or gel droplets that link together into long filamentous structures. In vitro experiments with the purified components of these aggregates suggests that aggregation is a form of phase separation, driven both by multivalent interactions between mRNAs and Whi3 and by polyQ associations between the Whi3 molecules [27]. Association with Whi3 creates mRNA rich patches near to nuclei and in the absence of Whi3 nuclei divide synchronously [18]. In a complex with a second low-complexity protein (Puf2) Whi3 performs a similar function by clustering formin transcripts at polarity sites [28]. Although relatively few examples of mRNA-protein complexes have been studied, the abundance of polyQ rich proteins in both fungal and animal proteomes [29] suggests that self-organized RNA-protein complexes may provide a general mechanism for localizing mRNAs to specific points of the cell.

Protein size For globular proteins, $d_{p}$ scales with sequence length raised to the 1/3-power, so even a twenty-five-fold difference in amino acid length (such 
as the difference between the smallest [200 a.a.] and largest [5000 a.a.] S. cere-

visiae proteins [30]) decreases $D$ only by a factor $25^{1 / 3}=2.9$. However in yeast cells proteins can also form very large aggregates: for example the heat shock protein Hsp104 induces damaged proteins to form large aggregates with typical diffusivities of $D=6 \times 10^{-4} \mu \mathrm{m}^{2} / \mathrm{s}$, and with half-lives on order of $\tau=1 \mathrm{hr}$ [31]. With these parameters, we expect Hsp104 induced protein aggregates to localize to cytosolic compartments of size: $\sqrt{D \tau}=2 \mu \mathrm{m}$. Similarly in yeast cells long lived proteins (LARPS) localize to parental cells and never enter bud cells; the two known cytoplasmic LARPS Hsp26 and Thr1 are found as macroaggregates [32]. Finally, mRNAs themselves diffuse more slowly than the proteins that they code for because nucleotides are much larger than the amino acids and because the mRNAs typically have ring-like rather than compact globular conformations, so their diameter is directly proportional to sequence length [33]. In particular, the diffusivity of cytoplasmic $\beta$-actin mRNA in mammalian cells is $D=0.1 \mu \mathrm{m}^{2} / \mathrm{s}$ [25], while the protein has a 700-fold larger diffusivity $(D=7.2$ $\mu \mathrm{m}^{2} / \mathrm{s}$ ) based on its diameter [34]. mRNAs often co-localize with their protein products [35] and because of their slower diffusion mRNAs rather than proteins may provide the templates for compartmentalizing the syncytium.

Altering bulk flow If the velocity of the fungal cytoplasm is $U$, then a macromolecule with half-life $\tau$ will be advected a distance of order $U \tau$ before being degraded. As shown in Figure 1B, cytoplasmic bulk flow in Neurospora crassa transports cytoplasmic proteins, including the cell cytoskeleton. This flow alters macromolecular distributions in two distinct senses: First, because of variations in velocity (known in physics as shear) across the hyphal crosssection from the fastest flow at the hyphal center to slowest flow near the hyphal walls, macromolecules and proteins are constantly rearranged with respect to each other (Fig 2A). Second, the flow of cytoplasm constantly advects organelles and proteins downstream from the sites of their production. For macromolecules whose targets are also flowing with the cytoplasm (for example cyclins that target free-flowing organelles like nuclei) local rearrangements within the cytoplasm are likely to be more important than the absolute position of the 
macromolecule within the syncytium. For macromolecules that target specific points in the hyphal wall (including secreted proteins or proteins involved in cell wall-remodeling) advection of macromolecules downstream is likely to be more important. We separately consider adaptations for controlling these two effects of flow:

Controlling rearrangements within the cytoplasm Typically fluids flowing in a narrow pipe adopt a parabolic velocity profile [36]: fluid sticks to the walls of the pipe and flows fastest in the center of the pipe. Because of this velocity gradient, particles introduced at different positions across the pipe are sheared out in the direction of low with time, with particles starting closest to the walls lagging particles that start near the center of the pipe (Fig. 2A). However, velocimetric measurements of bulk flow in real . N. crassa hyphae show little difference in velocity between cytoplasm near the hyphal walls and cytoplasm at the center of the hypha[37, 8]. The most likely explanation for the lack of shear is the viscoleastic nature of the cytoplasm: Filled with entangled polymers and large proteins, it flows more like toothpaste from a tube than water in a pipe. Polymeric liquids like toothpaste slip on rigid boundaries[38]. Due to the lack of velocity variation across the hypha, when we simulated the flow of nuclei and macromolecules in a mathematical model of a N. crassa hypha, we saw very little rearrangement (Fig. 2A). In particular when simulated macromolecules were followed for $30 \mathrm{~s}$, fewer than $5 \%$ changed the nucleus that they were most closely situated to, suggesting that interactions between macromolecules and organelles may be robust to flow in the hypha.

Controlling downstream advection In filamentous fungi, secreted proteins are translated within the Endoplasmic Reticulum (ER) [39]. In Aspergillus oryzae the distribution of ER can be mapped in BipA-GFP strains [40]: ER forms coronae around each nucleus and tubular structures around the periphery of the cell. Long peripheral ER tubules are likely to move less with cytoplasmic flow than nuclei, so may allow translation at fixed locations even within flowing hyphae. Additionally secretion in A. oryzae and A. niger seems to occur at sites (hyphal tips and septa $[41,42]$ ) in which flow is weakest. These regions of 
the hypha may allow secretion in the presence of bulk flow by sheltering protein traffic from the cytoplasmic stream.

Hyphal wall remodeling may require that proteins be targeted to the cell wall at regions that are not sheltered from flow; i.e regions away from hyphal tips and septa. In $N$. crassa motor driven transport allows nuclei to travel in a direction opposite to cell growth and bulk-cytoplasmic flow [43]. Although cellular microtubules are passively advected with the cytoplasm (Fig 1B and movie S1), the cell also contains cortical microtubules that are embedded in the plasma membrane [44], and we wondered whether nuclei and possibly other organelles could be trafficked on these microtubules even in fastest flowing hypha. Accordingly, we made a direct measurement of nuclear dynamics in N. crassa hyphae with fast $(i 5 \mu \mathrm{m} / \mathrm{s})$ flowing cytoplasm (following the method described in [8], see Fig. 2C and Movie S2). We found even in these fast flowing hypha a population of nuclei near the cell-membrane that were either stationary or moved against the direction of flow. Although we were not able to simultaneously image cortical microtubules, the counter-flowing nuclei were deformed with long tethers, consistent with the action of motor proteins applying focused forces on the nuclear membrane [45]. Restricting nuclei and potentially also macromolecules to a scaffold of microtubules at the cell membrane may create 205 a 2D surface on which cellular functions can be carried out even in the presence of flow in the bulk of the hypha.

Balancing diffusion and bulk-flow Although we have highlighted the role of cytoplasmic flow to disperse macromolecules within the cell, flow can also counteract diffusion to localize macromolecules. Proteins associated with secretion and polar growth are localized at hyphal tips: Macromolecules created at hyphal tips will tend to diffuse away from the tip, but can be confined by continual tipward flow of cytoplasm. The balance of diffusion and bulk flow localizes proteins to within a distance $D / U$ from the tip (Fig. 1C). For a small protein in a fast-growing $N$. crassa hypha $(U=1 \mu \mathrm{m} / \mathrm{s}, D=25 \mu \mathrm{m} / \mathrm{s})$ flow alone 215 will localize proteins to within a distance $d=25 \mu \mathrm{m}$ of the growing tip. Even weak cytoplasmic flow in slower growing syncytia can be augmented by septal 
walls. In ascomycete fungi, these walls contain small pores that are dynamically opened or closed by a specialized form of peroxisome called a Woronin body [46]. In older regions of the mycelium the pores are typically closed [23], and even small proteins like GFP can not pass through a closed pore; pore closure is thought to allow different compartments to function autonomously of each other [47]. Near hyphal tips, the pores are generally open [23, 47] allowing free passage of cytoplasm. However, examination of the physics that control macromolecular distribution shows that pores may allow only unidirectional flow of macromolecules: 1. passage through a small pore reduces protein diffusion; for a pore of diameter $d_{p}$ in a hypha of diameter $a$, the diffusivity for proteins traveling in either direction through a pore is reduced to $D_{p}=D d_{p}^{2} / a^{2}$ [47]. Since all of the cytoplasm flowing along the hypha must be focused to pass through the narrow pore; velocity within the pore is increased to $U_{p}=U a^{2} / d_{p}^{2}$. 230 In $A$. niger $d_{p} / a=0.04$ [47], so with a typical growth speed of $0.04 \mu \mathrm{m} / \mathrm{s}$, we predict $U_{p}=16.5 \mu \mathrm{m} / \mathrm{s}$ so that pores are unlikely to hinder transport of macromolecules in the direction of growth. However, proteins moving in the opposite direction must diffuse upstream, and their localization scale is $U_{p} / D_{p} \sim$ $D d_{p}^{4} / U a^{4}=1 \mathrm{~nm}$ for $A$. niger. Open septal pores may therefore provide one-way conduits; allowing proteins and mRNAs to be transported easily toward growing tips, but preventing them from diffusing in the opposite direction (Fig. 2B). Open pores may therefore act as barriers between different parts of syncytium and the large number of pore specific protein aggregates [48] is consistent with their acting as interfaces between functionally different compartments.

Life-time Diffusing macromolecules will diffuse a distance $\sqrt{D \tau}$, while advected macromolecules will be carried a distance $U \tau$, before being degraded (Fig. 1C). Both distances decrease as macromolecule life times decrease. Indeed, although cyclin half-lives are not known in filamentous fungi, in S. cerevisiae the half-life of $C L N 3$ mRNAs is only $2 \min [49]$. If their diffusivity is $D=0.2 \mu \mathrm{m}^{2} / \mathrm{s}$, then proteins will be localized to within $\sqrt{D \tau}=7 \mu \mathrm{m}$ of the nuclei from which they originate; i.e. shared only between this nucleus and one or two nearest neighbors. However, reducing mRNA lifetime while maintaining mRNA abun- 
dance means that more mRNAs need to be transcribed at greater cost to the cell.

\section{Discussion - An heterogeneous or homogeneous cellular interior?}

We have physically organized the known adaptations used by fungi to localize macromolecules at specific points in the cell and highlighted evidence that these macromolecules are controllably and heterogeneously patterned through the syncytium. At the same time, dispersal of macromolecules through a shared 255 cytoplasm endows fungi with tolerance for internal genetic diversity [7]. In addition to providing a source of phenotypic plasticity [17], shielding of deleterious mutations may allow more rapid adaptation to fungicides and new hosts. For example in Fusarium oxsporum, virulence effector genes seem to be concentrated in unstable genomic regions [50], while in Aspergillus nidulans ploidychanges accelerate adaptation to fungicides [51]. Although the direct necessity of chimerism is not proven in these cases, it is supported by studies of the basidiomycete species complex Rhizoctonia solani. Here the strain (AG8) with the largest host range produces multinucleate compartments, and bears signatures of hypermutation in many of the genes shared in common by its nuclei [52]. In addition to the interactions between fungus and host the balance of localization and dispersion of macromolecules creates complex population-level dynamics when the different nucleotypes harbored within the same syncytium. For example Neurospora tetrasperma contains two different nucleotypes and homokaryotic mycelia created from the two nucleotypes individually grow at different rates. Yet the two nucleotypes remain in stable but imbalanced proportions when harbored within a single mycelium [53]. If cyclins were not shared, then the proportions of the two nucleotypes would skew more and more toward the faster growing mating type over time. Conversely, if cyclins were freely dispersed then nucleotypes would be maintained in equal rather than imbalanced proportions. Fast cytoplasmic flows provide a mechanism for distributing different nucleotypes uniformly through the mycelial network [8], but physical model- 
ing suggests that controlled diffusion or cytoplasmic mixing of macromolecules between nuclei is also necessary. Uninucleate cells and bacteria encounter the problem of equipartitioning macromolecules between nuclei or cells only once in every cell-division cycle [54]. The ability of fungi to continuously solve this partitioning problem between genetically and epigenetically diverse nuclei may advance them as model systems for understanding how cells deal with genetic as well as environmental and physical sources of variation.

\section{Acknowledgements}

MR and PCH are supported by the NSF (DMS-1351860) and the Alfred P. Sloan Foundation. ASG is supported by the NIH (NIGMS R01GM081506) and the NSF (MCB-507511) 
Figure 1: Filamentous fungi create dynamic syncytia containing hundreds or even millions of nuclei sharing a single connected cytoplasm. (A) smFISH allows mRNA patterning to be directly measured in the shared cytoplasm of an Ashbya gossypii syncytium. (B) Fast cytoplasmic flow can disrupt macromolecule distributions by carrying cytoskeleton and nuclei downstream. Here we show microtubule distributions in two Neurospora crassa hyphae. Within a slow-flowing hypha GFP-labelled microtubules [55] provide a stable cytoskeleton (white arrow). In a fast-flowing hypha, the microtubules are advected with the cytoplasm (orange arrow). Scale bar: $10 \mu \mathrm{m}$. The interval between frames is $1.55 \mathrm{~s}$. (See also Movie S1). (C) Macromolecules (orange dots) are dispersed from the sites of their production (e.g. mRNAs from the nuclei that transcribe them) by a combination of diffusion and bulk cytoplasmic flow. Balancing diffusion, bulk flow and lifetime leads to different levels of macromolecule localization: $\sqrt{D \tau}$ if diffusion and degradation dominant, $U \tau$ is flow and degradation dominate and $D / U$ if diffusion and flow dominate. 
Figure 2: Multiple physical adaptations counteract the diffusion and flow of proteins. (A) We simulate the displacement of macromolecules (green) and nuclei (blue) after 30s of flow at $1 \mu \mathrm{m} / \mathrm{s}$, representing flow within a Neurospora crassa hypha. Upper panel: initial positions, middle panel: predicted displacement using parabolic velocity, lower panel: predicted displacement using measured velocity profile. Although organelles and macromolecules are all moved downstream, few rearrangements occur when the real velocity profile is used. (B) Open septal pores act as diffusion blocks limiting the flow or mRNAs and proteins, allowing different compartments to perform autonomous functions. Absent flow, there is hindered diffusion across the pore. However, in the presence of cytoplasmic flow, the pore can function like diode allowing proteins and mRNAs to freely travel in the direction of flow (here left to right), but preventing all transport in the opposite direction. (C) Nuclei can be held stationary, or even trafficked in the opposite direction to flow (green arrows), in a hypha with fast cytoplasmic flow (orange arrows show motion of nuclei that are traveling with the bulk flow) (see also Movie S2). We hypothesize that cortical microtubules may anchor macromolecules generally against cytoplasmic flow. Time elapsed between frames is 0.7s. See also Movie S2. 
[1] S. Vogel, Life in Moving Fluids: The Physical Biology of Flow, Princeton University Press, 1996.

[2] R. R. Lew, Mass flow and pressure-driven hyphal extension in Neurospora crassa, Microbiology 151 (8) (2005) 2685-2692.

[3] G. Steinberg, Hyphal growth: a tale of motors, lipids, and the Spitzenkörper, Eukaryot. Cell 6 (3) (2007) 351-360.

[4] C. Lang, S. Grava, T. van den Hoorn, R. Trimble, P. Philippsen, S. L. Jaspersen, Mobility, microtubule nucleation and structure of microtubuleorganizing centers in multinucleated hyphae of Ashbya gossypii, Mol. Biol. Cell 21 (1) (2010) 18-28.

[5] A. Walther, J. Wendland, Septation and cytokinesis in fungi, Fungal Genet. Biol. 40 (3) (2003) 187-196.

[6] N. L. Glass, K. Dementhon, Non-self recognition and programmed cell death in filamentous fungi, Curr. Opin. Microbiol. 9 (6) (2006) 553-558.

[7] * M. Roper, C. Ellison, J. W. Taylor, N. L. Glass, Nuclear and genome dynamics in multinucleate ascomycete fungi, Curr. Biol. 21 (18) (2011) R786-R793.

A recent review of nuclear heterogeneity in ascomycete fungi, and its role in mediating lateral gene transfer.

[8] * M. Roper, A. Simonin, P. C. Hickey, A. Leeder, N. L. Glass, Nuclear dynamics in a fungal chimera, P. Natl. Acad. Sci. USA 110 (32) (2013) 12875-12880.

Mathematical modeling and nuclear tracking shows that network flows in N. crassa syncytia are exquisitely optimized to mix nuclei and organelles. 
[9] R. Maheshwari, Nuclear behavior in fungal hyphae, FEMS Microbiol. Lett. 249 (1) (2005) 7-14.

[10] G. von Dassow, G. Schubiger, How an actin network might cause fountain streaming and nuclear migration in the syncytial Drosophila embryo., J. Cell Biol. 127 (6) (1994) 1637-1653.

[11] L. Carvalho, J. Stühmer, J. S. Bois, Y. Kalaidzidis, V. Lecaudey, C.-P. Heisenberg, Control of convergent yolk syncytial layer nuclear movement in zebrafish, Development 136 (8) (2009) 1305-1315.

[12] P. Gönczy, S. Pichler, M. Kirkham, A. A. Hyman, Cytoplasmic dynein is required for distinct aspects of MTOC positioning, including centrosome separation, in the one cell stage Caenorhabditis elegans embryo, J. Cell. Biol. 147 (1) (1999) 135-150.

$[13]$ * R. T. Johnson, P. N. Rao, Nucelo-cytoplasmic interactions in the achievement of nuclear synchrony in DNA synthesis and mitosis in multinucleate cells, Biol. Rev. 46 (1) (1971) 97-155.

A classic review of nuclear behavior in synthetically created syncytia.

[14] J. Demeter, S. E. Lee, J. Haver, T. Stearns, The DNA damage checkpoint signal in budding yeast is nuclear limited, Mol. Cell 6 (2000) 487-92.

[15] D. R. Nair, C. A. D’Ausilio, P. Occhipinti, M. E. Borsuk, A. S. Gladfelter, A conserved G1 regulatory circuit promotes asynchronous behavior of nuclei sharing a common cytoplasm, Cell Cycle 9 (18) (2010) 3795-3803.

[16] C. A. Anderson, U. Eser, T. Korndorf, M. E. Borsuk, J. M. Skotheim, A. S. Gladfelter, Nuclear repulsion enables division autonomy in a single cytoplasm, Curr. Biol. 23 (20) (2013) 1999-2010.

[17] * J. L. Jinks, Heterokaryosis: a system of adaptation in wild fungi, P. R. Soc. B 140 (898) (1952) 83-99. 
A classic work showing how dynamic control of nuclear ratios enables chimeric fungi to adapt to heterogenous environments

[18] * C. Lee, H. Zhang, A. E. Baker, P. Occhipinti, M. E. Borsuk, A. S. Gladfelter, Protein aggregation behavior regulates cyclin transcript localization and cell-cycle control, Dev. Cell 25 (6) (2013) 572-584.

smFISH imaging shows how the spatial distribution of cyclin transcripts, and nuclear autonomy, depends on the self-assembly of mRNA-protein aggregates.

[19] K. Zarnack, M. Feldbrügge, Microtubule-dependent mRNA transport in fungi, Eukaryot. Cell 9 (7) (2010) 982-990.

[20] I. Gonzalez, S. B. Buonomo, K. Nasmyth, U. von Ahsen, Ash1 mRNA localization in yeast involves multiple secondary structural elements and Ash1 protein translation, Curr. Biol. 9 (6) (1999) 337-340.

[21] * A. J. Rodriguez, K. Czaplinski, J. S. Condeelis, R. H. Singer, Mechanisms and cellular roles of local protein synthesis in mammalian cells, Curr. Opin. Cell Biol. 20 (2) (2008) 144-149.

A review of mRNA localization and transportation mechanisms in mammalian cells, focusing on the role of the cytoskeleton.

[22] E. Vollmeister, K. Schipper, M. Feldbrügge, Microtubule-dependent mRNA transport in the model microorganism Ustilago maydis, RNA Biol. 9 (3) (2012) 261-268.

[23] A. Trinci, Influence of the width of the peripheral growth zone on the radial growth rate of fungal colonies on solid media, J. Gen. Microbiol. 67 (3) (1971) 325-344.

[24] P. Nelson, Biological Physics, 2nd Edition, Freeman, 2004.

[25] * D. Fusco, N. Accornero, B. Lavoie, S. M. Shenoy, J.-M. Blanchard, R. H. Singer, E. Bertrand, Single mRNA molecules demonstrate probabilistic movement in living mammalian cells, Curr. Biol. 13 (2003) 161-7. 


\section{Direct imaging in live mammalian cells shows both corralling and diffusion of labelled cytoplasmic mRNAs.}

[26] E. Gari, T. Volpe, H. Wang, C. Gallego, B. Futcher, M. Aldea, Whi3 binds the mRNA of the G1 cyclin CLN3 to modulate cell fate in budding yeast, Genes Dev. 15 (2001) 2803-8.

[27] P. Li, S. Banjade, H.-C. Cheng, S. Kim, B. Chen, L. Guo, M. Llaguno, J. V. Hollingsworth, D. S. King, S. F. Banani, et al., Phase transitions in the assembly of multivalent signalling proteins, Nature 483 (7389) (2012) 336-340.

[28] C. Lee, P. Occhipinti, A. S. Gladfelter, PolyQ-dependent RNA-protein assemblies control symmetry breaking, J. Cell Biol. 208 (5) (2015) 533544.

[29] M. H. Schaefer, E. E. Wanker, M. A. Andrade-Navarro, Evolution and function of $\mathrm{CAG} /$ polyglutamine repeats in protein-protein interaction networks, Nucleic Acids Res. 40 (10) (2012) 4273-4287.

[30] J. Warringer, A. Blomberg, Evolutionary constraints on yeast protein size, BMC Evol. Biol. 6 (1) (2006) 61.

[31] C. Zhou, B. D. Slaughter, J. R. Unruh, A. Eldakak, B. Rubinstein, R. Li, Motility and segregation of Hsp104-associated protein aggregates in budding yeast, Cell 147 (2011) 1186-96.

[32] N. H. Thayer, C. K. Leverich, M. P. Fitzgibbon, Z. W. Nelson, K. A. Henderson, P. R. Gafken, J. J. Hsu, D. E. Gottschling, Identification of longlived proteins retained in cells undergoing repeated asymmetric divisions, P. Natl. Acad. Sci. USA 111 (39) (2014) 14019-14026.

[33] R. Milo, P. Jorgensen, U. Moran, G. Weber, M. Springer, BioNumbersthe database of key numbers in molecular and cell biology, Nucleic Acids Res. 38 (suppl 1) (2010) D750-D753. 
[34] J. Xu, W. H. Schwarz, J. A. Käs, T. P. Stossel, P. A. Janmey, T. D. Pollard, Mechanical properties of actin filament networks depend on preparation, polymerization conditions, and storage of actin monomers, Biophys. J. 74 (5) (1998) 2731-2740.

[35] E. Lécuyer, H. Yoshida, N. Parthasarathy, C. Alm, T. Babak, T. Cerovina, T. R. Hughes, P. Tomancak, H. M. Krause, Global analysis of mRNA localization reveals a prominent role in organizing cellular architecture and function, Cell 131 (1) (2007) 174-187.

[36] G. K. Batchelor, An Introduction to Fluid Dynamics, Cambridge University Press, 2000.

[37] A. Abadeh, R. R. Lew, Mass flow and velocity profiles in Neurospora hyphae: partial plug flow dominates intra-hyphal transport, Microbiology 159 (Pt 11) (2013) 2386-2394.

[38] P.-G. De Gennes, Wetting: statics and dynamics, Rev. Mod. Phys. 57 (3) (1985) 827.

[39] J.-y. Shoji, M. Arioka, K. Kitamoto, Dissecting cellular components of the secretory pathway in filamentous fungi: insights into their application for protein production, Biotechnol. Lett. 30 (1) (2008) 7-14.

[40] J.-i. Maruyama, S. Kikuchi, K. Kitamoto, Differential distribution of the endoplasmic reticulum network as visualized by the BipA-EGFP fusion protein in hyphal compartments across the septum of the filamentous fungus, Aspergillus oryzae, Fungal Genet. Biol. 43 (9) (2006) 642-654.

[41] C. L. Gordon, V. Khalaj, A. F. Ram, D. B. Archer, J. L. Brookman, A. P. Trinci, D. J. Jeenes, J. H. Doonan, B. Wells, P. J. Punt, et al., Glucoamylase::green fluorescent protein fusions to monitor protein secretion in Aspergillus niger, Microbiology 146 (2) (2000) 415-426.

[42] K. Masai, J.-I. Maruyama, H. Nakajima, K. Kitamoto, In vivo visualization of the distribution of a secretory protein in Aspergillus oryzae hyphae using 
the RntA-EGFP fusion protein, Biosci. Biotech. Bioch. 67 (2) (2003) 455459.

[43] S. L. Ramos-García, R. W. Roberson, M. Freitag, S. Bartnicki-García, R. R. Mouriño-Pérez, Cytoplasmic bulk flow propels nuclei in mature hyphae of Neurospora crassa, Eukaryot. Cell 8 (12) (2009) 1880-1890.

[44] M. Uchida, R. R. Mouriño Pérez, R. W. Roberson, Live-cell imaging of microtubule dynamics in hyphae of Neurospora crassa, in: A. Sharon (Ed.), Molecular and Cell Biology Methods for Fungi, Vol. 638 of Methods in Molecular Biology, Springer, Berlin, 2010, pp. 259-268.

[45] I. Derényi, G. Koster, M. Van Duijn, A. Czövek, M. Dogterom, J. Prost, Membrane nanotubes, in: H. Linke, A. Månsson (Eds.), Controlled Nanoscale Motion, Vol. 711 of Lecture Notes in Physics, Springer, Berlin, 2007, pp. 141-159.

[46] G. Jedd, N.-H. Chua, A new self-assembled peroxisomal vesicle required for efficient resealing of the plasma membrane, Nature Cell Biology 2 (4) (2000) 226-231.

[47] * R.-J. Bleichrodt, M. Hulsman, H. A. Wösten, M. J. Reinders, Switching from a unicellular to multicellular organization in an Aspergillus niger hypha, mBio 6 (2) (2015) e00111-15.

Direct measurement of the dynamics of protein passage through the septal pores of a living filamentous fungus.

[48] * J. Lai, C. H. Koh, M. Tjota, L. Pieuchot, V. Raman, K. B. Chandrababu, D. Yang, L. Wong, G. Jedd, Intrinsically disordered proteins aggregate at fungal cell-to-cell channels and regulate intercellular connectivity, P. Natl. Acad. Sci. USA 109 (39) (2012) 15781-15786.

Mass-spectroscopy and bioinformatics reveal that there are many intrinsically disordered proteins functioning at septal pores. 
[49] Y. Cai, B. Futcher, Effects of the yeast RNA-binding protein Whi3 on the half-life and abundance of CLN3 mRNA and other targets, PLoS ONE 8 (12) (2013) e84630.

[50] M. Rep, H. C. Kistler, The genomic organization of plant pathogenicity in Fusarium species, Curr. Opin. Plant Biol. 13 (4) (2010) 420-426.

[51] S. E. Schoustra, A. J. Debets, M. Slakhorst, R. F. Hoekstra, Mitotic recombination accelerates adaptation in the fungus Aspergillus nidulans, PLoS Genet. 3 (4) (2007) e68-e68.

[52] J. Hane, J. Anderson, A. Williams, J. Sperschneider, K. Singh, Genome sequencing and comparative genomics of the broad host-range pathogen Rhizoctonia solani AG8., PLoS genetics 10 (5) (2014) e1004281.

[53] * N. Samils, J. Oliva, H. Johannesson, Nuclear interactions in a heterokaryon: insight from the model Neurospora tetrasperma, P. R. Soc. B 281 (1786) (2014) 20140084.

Direct measurement show that in a hermaphroditic fungus proportions of nucleotypes are controlled through its life-cycle.

[54] I. Lestas, G. Vinnicombe, J. Paulsson, Fundamental limits on the suppression of molecular fluctuations, Nature 467 (7312) (2010) 174-178.

[55] M. Freitag, P. C. Hickey, N. B. Raju, E. U. Selker, N. D. Read, GFP as a tool to analyze the organization, dynamics and function of nuclei and microtubules in Neurospora crassa, Fungal Genet. Biol. 41 (10) (2004) 897910. 

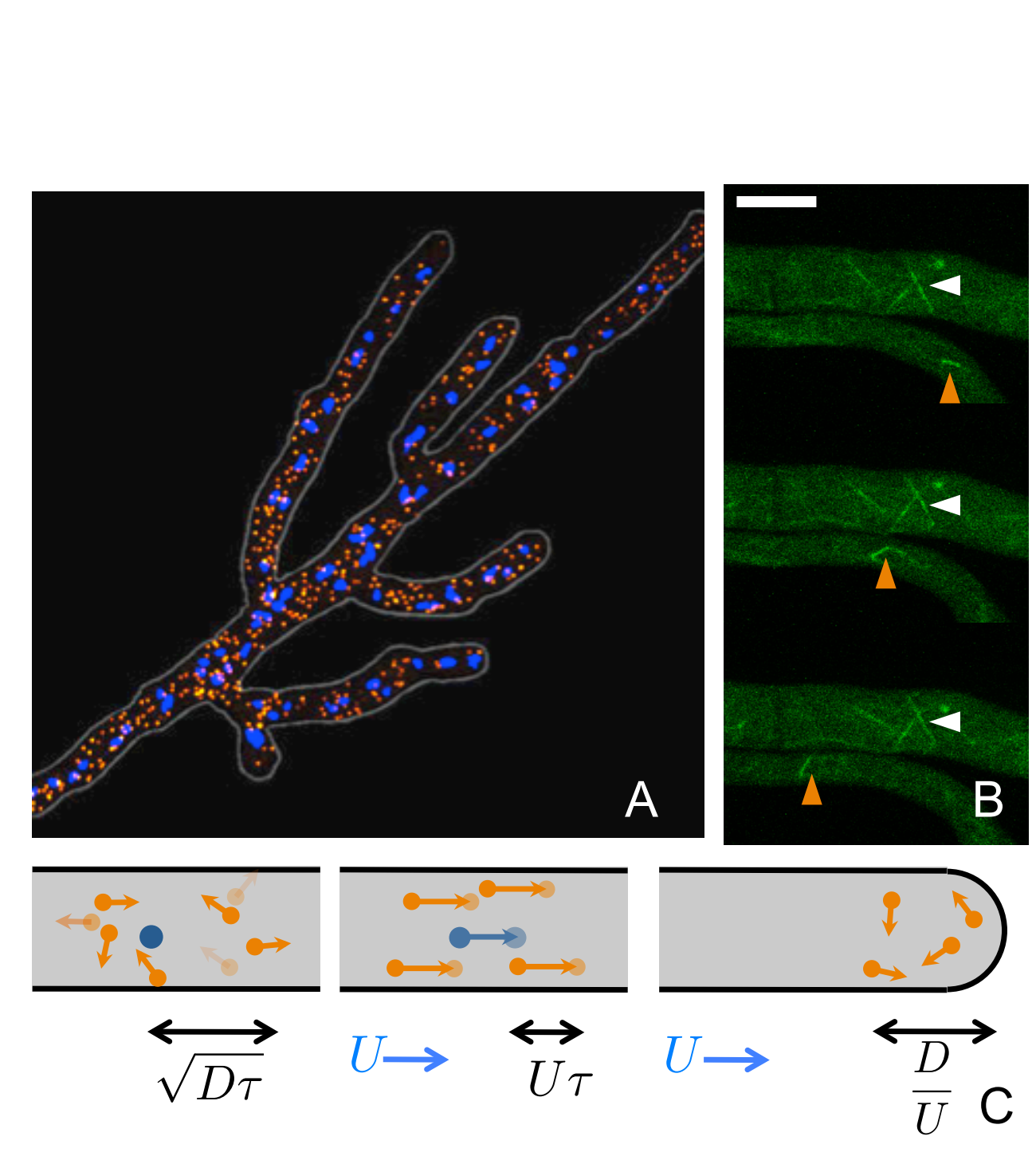

Figure 1.

.

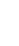




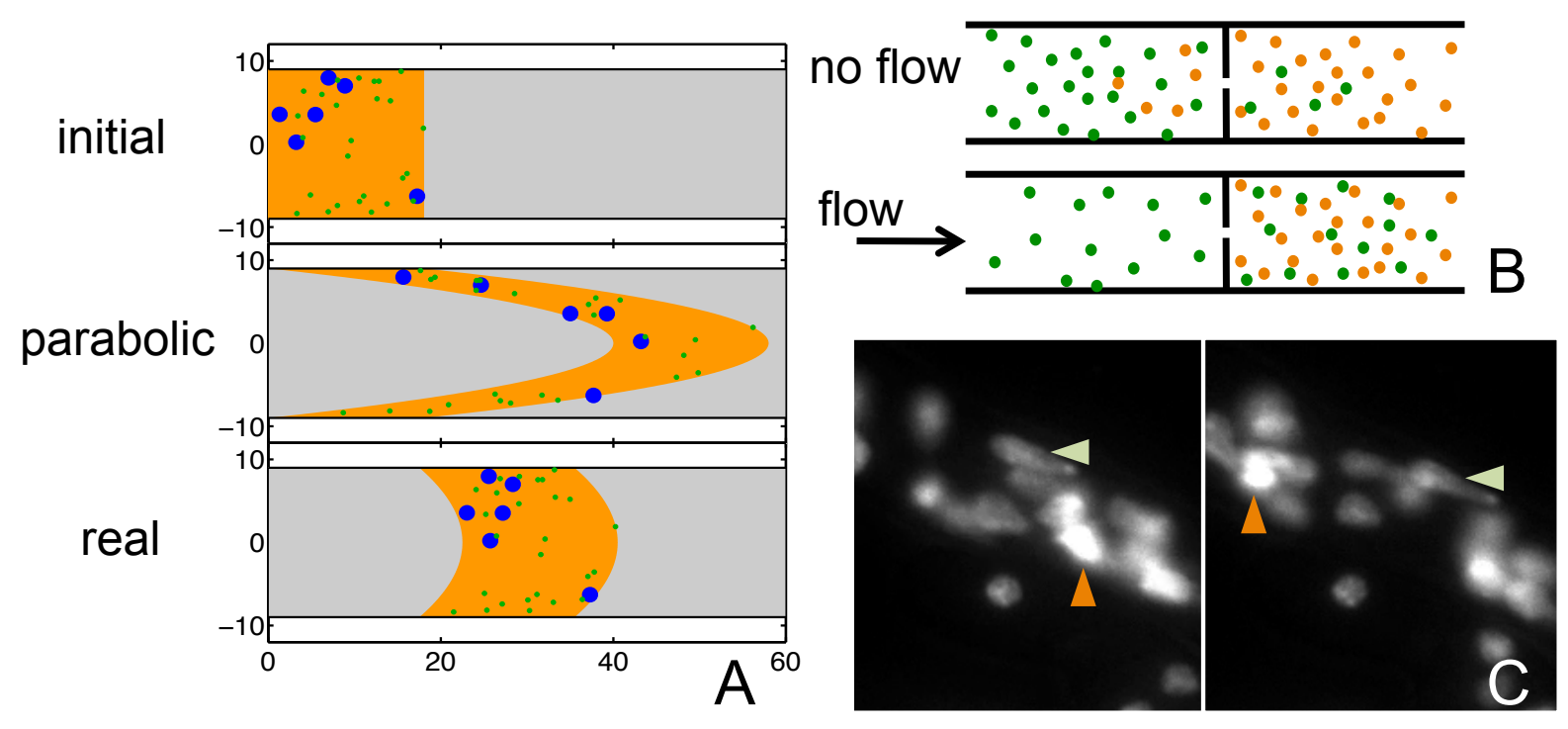

Figure 2. 